\title{
Compensation of Ultrasound Attenuation in Photoacoustic Imaging
}

\author{
P. Burgholzer ${ }^{1,2}$, H. Roitner ${ }^{1,2}$, J. Bauer-Marschallinger ${ }^{1,2}$, \\ H. Grün ${ }^{2}$, T. Berer ${ }^{1,2}$ and G. Paltauf ${ }^{3}$ \\ ${ }^{1}$ Christian Doppler Laboratory for Photoacoustic Imaging and Laser Ultrasonics, \\ ${ }^{2}$ Research Center for Non Destructive Testing (RECENDT), \\ ${ }^{3}$ Institute of Physics, Karl-Franzens-University Graz \\ Austria
}

\section{Introduction}

Photoacoustic imaging is a non-destructive method to obtain information about the distribution of optically absorbing structures inside a semitransparent medium. It is based on thermoelastic generation of ultrasonic waves by the absorption of a short laser pulse inside the sample. From the ultrasonic waves measured outside the object, the interior distribution of absorbed energy is reconstructed. The ultrasonic waves, which transport information from the interior to the surface of the sample, are scattered or absorbed to a certain extent by dissipative processes. The scope of this work is to quantify the information loss which is equal to the entropy production during these dissipative processes and thereby to give a principle limit for the spatial resolution which can be gained in photoacoustic imaging. This theoretical limit is compared to experimental data. In this book chapter stateof-the-art methods for modeling ultrasonic wave propagation in the case of attenuating media are described. From these models strategies for compensating ultrasound attenuation are derived which may be combined with well-known reconstruction algorithms from the non-attenuating case for photoacoustic imaging.

Section 2 gives a short description of photoacoustic imaging, especially photoacoustic tomography, and the available image reconstruction algorithms to reconstruct the interior structure from the detected ultrasound signal at the sample surface. Beside small point-like detectors also large detectors, so called integrating detectors are used for photoacoustic tomography. The latter ones require different image reconstruction algorithms. Spatial resolution is an essential issue for any imaging method. Therefore we describe the influencing factors of the resolution in photoacoustic tomography.

Section 3 is dedicated to acoustic attenuation. The spatial resolution in photoacoustic imaging is limited by the acoustic bandwidth. To resolve small objects shorter wavelengths with higher frequencies are necessary. For such high frequencies, however, the acoustic attenuation increases. This effect is usually ignored in photoacoustic image reconstruction but as small objects or structures generate high frequency components it limits the minimum detectable size, hence the resolution. Several models for acoustic attenuation, especially used for ultrasound propagation in biological tissue, are compared with experimental data. 
Section 4 describes two different attempts to compensate this acoustic attenuation: either to include the compensation directly in the image reconstruction, e. g. in a modified time reversal method, or to calculate first the acoustic signal without attenuation from the measured attenuated signal and then perform the conventional photoacoustic image reconstruction. As any compensation of acoustic attenuation is mathematically an ill-posed problem both methods need regularization to prevent small measurement fluctuations from growing infinitely high in the reconstructed image. The possible degree of compensation depends on the size of these fluctuations. On the other hand acoustic attenuation is a dissipative process that causes entropy production equal to a loss of information, which cannot be compensated by any compensation algorithm. Therefore one can use the entropy production caused by acoustic attenuation to determine the minimal fluctuations in the measurement data, which turn out to be equal to thermal fluctuations. In statistical physics this fact is well known as the fluctuation-dissipation theorem, but the information theoretical background as a starting point to derive this theorem was not mentioned before in the literature.

Section 5 uses stochastic processes to understand theoretically how information can be lost and its connection to entropy production. Therefore, the measured pressure signal is treated as a random variable with a certain mean value as a function of time and certain fluctuations around that value. First for the simple model of a damped harmonic oscillator, it is shown how information is lost during a dissipative process and to what extent we can reconstruct the original information after some time. Then attenuated acoustic waves can be treated in a similar way: the spatial Fourier transform of the pressure wave can be described by a similar stochastic process as the damped harmonic oscillator - only in a higher dimension. Each wave vector is represented by a damped oscillator of different frequency.

Thinking about acoustic attenuation as a stochastic process helps to understand how entropy production and loss of information "work" on a microscopic scale. Beside a better theoretical insight the stochastic view on the acoustic wave answers a very important question: which is the best compensation method and the corresponding practicable spatial resolution in photoacoustic imaging? This question can be answered without taking fluctuations on a microscopic scale into account: the entropy production, which can be calculated from macroscopic mean values, is set equal to the information loss.

\section{Photoacoustic imaging}

In 1880, Alexander Graham Bell discovered that pulsed light striking a solid substrate can produce a sound wave, a phenomenon called the photoacoustic effect (Bell, 1880). Practical imaging methods based on this effect have been developed and reported the last decade (Xu \& Wang, 2006). Today, photoacoustic imaging, which is also referred to as optoacoustic imaging or, when using microwaves instead of light for excitation, as thermoacoustic imaging, is attracting intense interest for cross-sectional or three-dimensional imaging in biomedicine. In photoacoustic imaging, short laser pulses are fired at a sample and the absorbed energy causes local heating (Fig. 1). This heating causes thermoelastic expansion and thereby generation of broadband elastic pressure waves (ultrasound) which can be detected outside the sample, for example by a piezoelectric device or by an optical detector. Two methods are used for photoacoustic imaging: photoacoustic microscopy uses focused ultrasonic detectors and the sample is imaged by scanning the focus through the sample. In photoacoustic tomography (PAT) an unfocused detector is used which detects the pressure from the 
ultrasound wave arriving from all different locations of the source. A map or "image" of the photo-generated pressure distribution in the sample can be made by collecting the ultrasound at many different locations and processing it using a suitable algorithm e.g. by a filtered backprojection algorithm or by a time reversal algorithm.

Only if the pulse is short enough, thermal expansion causes a pressure rise proportional to the locally absorbed energy density. Short enough means that the pressure wave does not "run out" of the smallest structure which should be resolved in the photoacoustic image during the pulse time. This so called "stress confinement" is therefore fulfilled if the sound velocity multiplied by the pulse time of the laser is small compared to the spatial resolution one wants to achieve in imaging. Another constraint is the "thermal confinement" which is fulfilled if the heat induced in a structure by the absorbed laser pulse does not diffuse out of this structure during the time of the laser pulse. As heat diffusion is usually slower than the propagation of sound the thermal confinement is fulfilled if stress confinement is fulfilled.

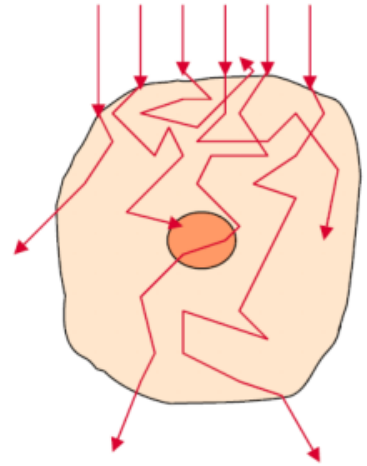

(a)

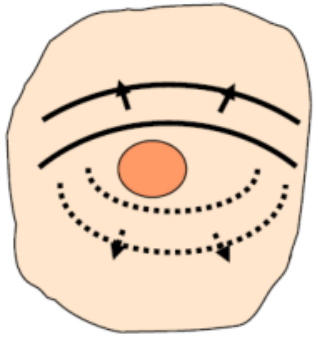

(b)

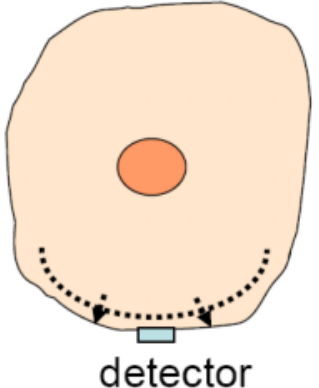

(c)

Fig. 1. Photoacoustic Imaging - the spatial resolution is determined by excitation, propagation, and detection of the acoustic wave. (a) Thermoelastic generation of acoustic wave by laser light (arrows indicate scattered photons): excited pressure is proportional to the absorbed optical energy density, if laser pulse is short enough to satisfy thermal and stress confinement. (b) Propagation of ultrasonic wave to sample surface: frequency dependent acoustic attenuation causes entropy production and therefore a loss of information. (c) Detection of ultrasonic wave: bandwidth and size of detector limits spatial resolution

Any photons, either unscattered or scattered (see arrows in Fig. 1), contribute to the absorbed energy as long as the photon excitation is relaxed thermally. Therefore PAT visualizes the product of the optical absorption distribution and the local light fluence.

Using a Nd:YAG laser and an optical parametric oscillator (OPO) light pulses from the infrared to the visible regime can be selected with a repetition rate from $10 \mathrm{~Hz}$ up to $100 \mathrm{~Hz}$. Some high speed PAT systems can even go up to $1000 \mathrm{~Hz}$. The pulse duration in the nanosecond range enables a theoretical resolution of several microns in tissue (sound velocity similar to water at approx. $1500 \mathrm{~m} / \mathrm{s}$ ). For biomedical applications the light energy should not exceed $20 \mathrm{~mJ} / \mathrm{cm}^{2}$ in the visible spectral range and $100 \mathrm{~mJ} / \mathrm{cm}^{2}$ in the near infrared light. 
If acoustic attenuation and shear waves (in liquid and soft tissue) are neglected, the acoustic pressure $p$ as a function of time and space obeys the equation

$$
\Delta p(\mathbf{r}, t)-\frac{1}{c^{2}} \frac{\partial^{2}}{\partial t^{2}} p(\mathbf{r}, t)=-\frac{\beta}{C_{p}} \frac{\partial}{\partial t} \mathrm{H}(\mathbf{r}, t)
$$

where $\Delta$ is the three-dimensional Laplace operator, $c$ the sound velocity, $\beta$ the thermal expansion coefficient, $C_{p}$ the specific heat capacity and $H(\mathbf{r}, t)$ the deposited energy per time and volume ("heating function") caused by the absorption of the electromagnetic radiation in the sample.

For short electromagnetic pulses $H(\mathbf{r}, t)=A(\mathbf{r}) \cdot \delta(t)$, where $A(\mathbf{r})$ is the energy density of the absorbed electromagnetic radiation and $\delta(t)$ the Dirac delta function. Then the acoustic pressure $p(\mathbf{r}, t)$ solves the homogeneous scalar wave equation with the initial conditions $p(\mathbf{r}, 0)=p_{0}(\mathbf{r})=\beta c^{2} / C_{p} \cdot A(\mathbf{r}) \equiv \Gamma \cdot A(\mathbf{r})$ and $\partial / \partial t p(\mathbf{r}, 0)=0$. The initial pressure $p_{0}$ at time $t=0$ is therefore directly proportional to the absorbed energy density $A$ with the dimensionless constant $\Gamma$, the Grüneisen coefficient.

As shown in Fig. 1 (c) bandwidth and size of the detectors for collecting the ultrasound signals are important for the resolution of this imaging modality. A photoacoustically generated ultrasound signal is a broadband signal and contains frequencies in the range from kilohertz up to a few megahertz. Conventional point like piezo elements such as known from arrays for medical ultrasonic imaging have their maximum sensitivity close to their center frequency and can detect frequencies only within a certain bandwidth around this frequency. Therefore high frequencies are not detected which correspond to small structures and are necessary for image reconstruction with a high spatial resolution. Other approaches are necessary for high resolution photoacoustic imaging. A hydrophone could be one solution (Wang, 2008), or the utilization of optical point like detection as demonstrated e.g. by (Zhang et al., 2009) or (Berer et al., 2010).

Point like detectors show a limit in achievable resolution by their size. The smaller the point detector the better is the spatial resolution. Unfortunately thermal and other fluctuations increase for a smaller detector, which results in a reduction of resolution. A totally different approach is the use of so called integrating detectors which are at least in one dimension larger than the object. This way the drawback of finite dimensions of point like detectors can be overcome. Such an integrating detector for photoacoustic imaging was introduced by (Haltmeier et al, 2004). They showed the mathematical proof of integrating area and line detectors and introduced new reconstruction methods which are necessary when using such a detector. First measurements using an integrating detector were shown by (Burgholzer et al., 2005). The first integrating detector was an area detector which was bigger than the object in two dimensions. For sufficient data for 3D image reconstruction the area detector had to be scanned around the object tangential to the surface of a sphere. This detector movement is difficult to realize. Hence the idea of the integrating line detector was developed. A fragmentation of the area detector into an array of line detectors results in an easier setup with only one rotation axis for the object and a linear motion of the integrating line detector (Fig. 2).

An integrating line detector is a line which has at least a length $\sqrt{8}{ }^{*} D$, where $D$ is the diameter of a circle enclosing the sample and tangentially touching the line detector (Haltmeier et al., 2004). The line detector integrates the pressure along the line on a 
cylindrical surface with the radius $c \cdot t$ where $c$ is the speed of sound in the medium and $t$ the time. Thus, integrating line detectors arranged in an array around the sample, e.g. in a circle, measure projection images of the object in a first measurement and reconstruction step. By rotating the sample and measuring such projection images from different angles it is possible to reconstruct a 3D image (Fig. 2). Three dimensional image reconstruction from a set of projection images requires only the application of the inverse Radon transform. Therefore $3 \mathrm{D}$ imaging using integrating line detectors is not computationally intensive compared with other algorithms which reconstruct a 3D image from a set of signals acquired from point like detectors.

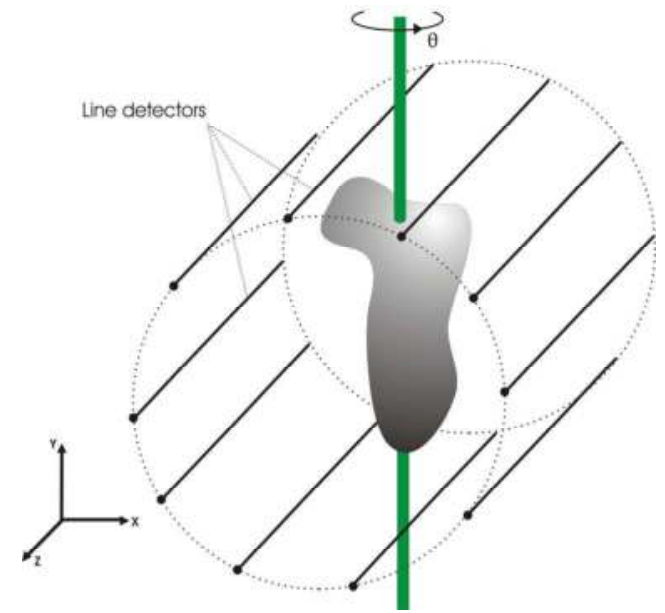

Fig. 2. Line detectors around a sample rotated on one axis. Either one line detector is scanning around the object or a detector array is used

Since the first measurement results from (Burgholzer et al, 2005) the integrating line detector was further improved in sensitivity and spatial resolution. Several types of such line detectors were implemented. One premature approach was a line made of PVDF (piezo foil) which provides high sensitivity but with the drawback of directivity. The next consequential step was an optical line detector realized by an interferometer. A laser beam that is part of an interferometer measures variations of the refractive index induced by the acoustic pressure (elasto-optic effect) (Paltauf et al., 2006). Optical detectors offer a broadband characteristic and due to the circular shape of a laser beam or light guiding fiber there is no such directivity like when using a film of piezo foil. Two main approaches can be distinguished: free-beam implementations and the use of fiber-based interferometers. Independent of the realization different types of the interferometer can be used, e.g. a MachZehnder or a Fabry-Perot interferometer which is in general more sensitive than the first one. (Paltauf et al., 2006) presented measurements using a free-beam Mach-Zehnder interferometer. They used a focused laser beam as line detector. When placing the object next to the beam waist of the focused laser beam the best spatial resolution due to the smallest beam diameter could be achieved (Paltauf et al., 2008).

(Grün et al, 2010) implemented fiber-based line detectors. The advantages of fiber-based line detectors are the easy handling and the small and constant beam diameter in the fiber. A small diameter of the laser beam is necessary for a good spatial resolution. The smaller the 
diameter of the detecting part the better is the spatial resolution. A typical single mode fiber for near infrared has a core diameter of 9 microns; single mode fibers for the visible range of detection wavelength have typically about 6 microns. Due to the constant diameter along the whole line detector this type of integrating line detector is dedicated for the imaging of big samples. After first implementations of a Mach-Zehnder and a Fabry-Perot interferometer in glass fibers now polymer fibers are used. Due to the much better impedance matching of polymer fibers to the surrounding water, their sensitivity is higher than for glass fibers, where approximately $2 / 3$ of the incoming signal is reflected before reaching the core (Grün et al., 2010). Furthermore the Young's modulus is much lower in polymer fibers than in glass fibers for which reason the deformation of a polymer fiber is bigger than of a glass fiber applying the same pressure wave. As the strain optic coefficients are in the same order, this results in an enhanced change of refractive index and thus to higher signal amplitudes in the polymer fiber (Kiesel et al., 2007).

(Nuster et al, 2009) did a comparison of the different implementations of an integrating line detector. At this stage of development the free-beam Mach-Zehnder interferometer was the most sensitive integrating line detector. But these measurements showed some new approaches how the fiber-based line detector could be made more sensitive, e.g. by building up a Fabry-Perot interferometer in a single mode polymer fiber.

The next step after developing a sensitive line detector, no matter of which approach is the most sensitive one, is the creation of an array of many integrating line detectors, e.g. 200 detectors arranged in a curve around the sample. This way one could acquire all data for a projection image within one excitation pulse of the laser.

\section{Acoustic attenuation}

The imaging resolution in photoacoustic imaging is limited by the acoustic bandwidth and therefore by the laser pulse duration as mentioned above, but also by the attenuation of the acoustic wave on its way to the sample surface, and finally by the bandwidth and size of the ultrasonic transducer. The acoustic attenuation can be substantial for high frequencies. This effect is usually ignored in reconstruction algorithms but can have a strong impact on the resolution of small objects or structures within objects. Stokes could already show in 1845 that for liquids with low viscosity, such as water, the acoustical absorption increases by the square of the frequency (Stokes, 1845). If we do not take acoustic attenuation for photoacoustic image reconstruction into account, especially small structures (corresponding to shorter wavelengths and therefore higher frequencies) appear blurred (La Riviere et al., 2006). To what extent this blurring can be compensated by regularization methods (as performed by (La Riviere et al., 2006)) and how much information is lost due the irreversibility of attenuation is investigated in this chapter.

For thin layers (1D), small cylinders (2D), and small spherical inclusions (3D) the effect of attenuation is simulated and experimental results for several types of tissue are given. For photoacoustic tomography a new description of attenuation seems to be useful: like for a standing wave in a resonator the wave number is real but the frequency is complex. The complex part of the frequency is the damping in time. The resulting pressure wave as the solution of the wave equation is of course the same as by decomposing into plane waves with complex wave number. But with the complex frequency description acoustic attenuation can be included in all "k-space" methods well known in photoacoustic tomography just by introducing a factor describing the exponential decay in time (Roitner \& Burgholzer, 2011). 
Acoustic attenuation is an irreversible process and therefore the wave equation is not invariant to time reversal. Several important reasons for acoustic attenuation have been reported: viscosity, heat conduction, relaxation processes and chemical reactions. Stokes derived the scalar wave equation

$$
\Delta p-\frac{1}{c^{2}} \frac{\partial^{2} p}{\partial t^{2}}+\tau \Delta \frac{\partial p}{\partial t}=0
$$

under the assumption of adiabatic conditions (thus neglecting the loss due to heat conduction) (Stokes, 1845). This equation can also be found in (Shutilov, 1988) and can generally be derived from a relaxation behavior of pressure and density (Royer \& Dieulesaint, 2000), where the density change follows the pressure change after a relaxation time $\tau$. If $\tau$ is further expressed in terms of viscosity and specific heat, this equation is also known as the thermoviscous equation. Eq. (2) describes acoustic attenuation which is approximately proportional to the square of the frequency. Other wave equations can describe a more general power law frequency dependence of the attenuation of the form $\alpha(\omega)=\alpha_{0} \cdot|\omega|^{y}(0<\mathrm{y}<3)$. (Szabo, 1994) has suggested adding the loss term $L(t) * p(\mathbf{r}, t)$ to the wave equation in order to account for such attenuation behavior:

$$
\Delta p(\mathbf{r}, t)-\frac{1}{c_{0}^{2}} \frac{\partial^{2}}{\partial t^{2}} p(\mathbf{r}, t)+L(t) * p(\mathbf{r}, t)=0
$$

Other models for acoustic wave propagation in acoustic media have been proposed also by (Nachman et al., 1990) and (Treeby \& Cox, 2010).

\section{Measurement and simulation of broadband acoustic attenuation:}

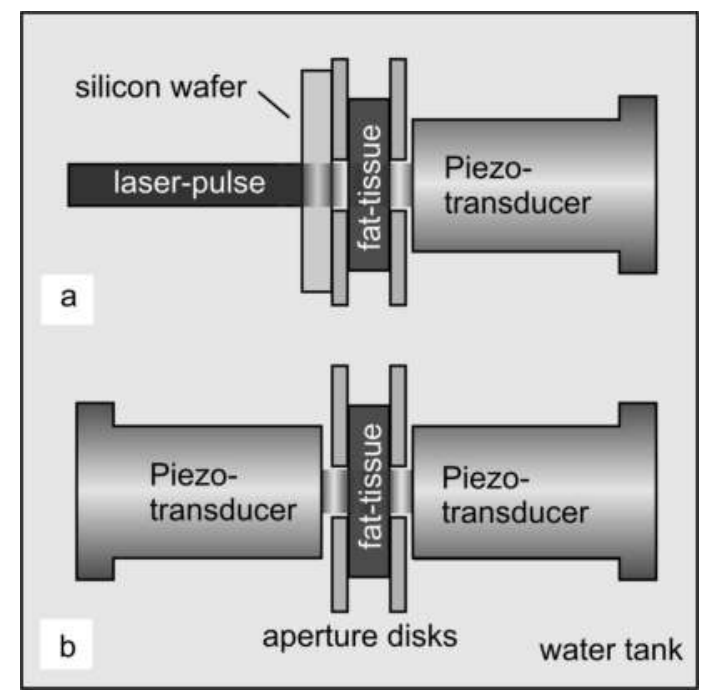

Fig. 3. Experimental set-up to measure broadband high frequency acoustic attenuation in tissue with ultrasound generated by short laser pulses (a) and by piezoelectric transducers (b). In both cases a piezoelectric transducer receives the ultrasonic signals 
Fig. 3 shows the set up to determine frequency dependent acoustic attenuation in tissue by a single transmission experiment. High frequency ultrasonic pulses are either generated by a pulse laser (pulse duration $6 \mathrm{~ns}$ ) heating up the surface of a silicon wafer (Fig. 3a) or by a piezoelectric transducer (Fig. 3b). The resulting planar-like ultrasound waves propagate through a fat tissue with varying thickness and through distilled water as a coupling medium to a piezoelectric transducer. The ultrasonic attenuation $\alpha$ is determined by comparing transmission measurements of the investigated samples and distilled water. In Fig. 4 Fourier transformed attenuation results for subcutaneous fat of pig, human blood and olive oil are shown. A power law $\alpha(f)=\alpha_{0} \cdot f^{y}$ can be applied to those substances, where $\mathrm{f}$ denotes the frequency (Bauer-Marschallinger et al., 2011).

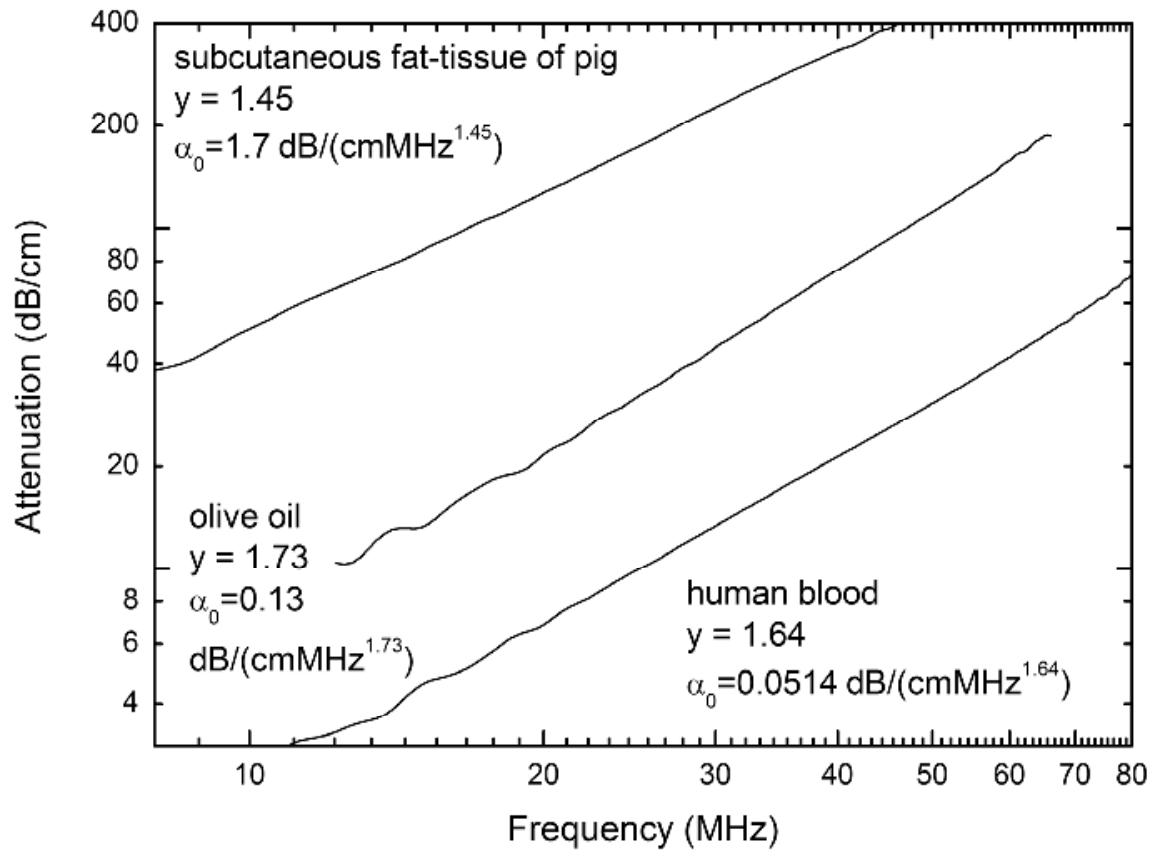

Fig. 4. Attenuation as a function of frequency for three biological substances (double logarithmic scale)

The attenuated planar ultrasound waveform can be calculated by a number of available simulation methods. In a comprehensive study (Roitner et al., 2011) we compared measured waveforms for two fat thicknesses $(3.2 \mathrm{~mm}$ and $6.2 \mathrm{~mm})$ to simulated waveforms obtained with three current simulation methods. The methods using the Matlab toolbox by (Treeby \& Cox, 2010b) or the relation (4) by (LaRiviere et al., 2006) rely on a frequency power law absorption and are described in detail below. A third method by (Nachman et al., 1990) assumes multiple molecular relaxation processes as the cause of attenuation. These processes are characterized by their contributions $\kappa_{n}(n=1 \ldots N)$ to the isothermal compressibility of the tissue and the relaxation times $\tau_{n}(n=1 \ldots N)$ of their vibrational 
energies. With these parameters a complex wave number is obtained involving a generalized compressibility $\kappa(t)=\kappa_{\infty} \delta(t)+\sum_{n=1}^{N} \frac{\kappa_{n}}{\tau_{n}} \exp \left(-t / \tau_{n}\right) H(t)$ where $\kappa_{\infty}=\chi-\sum_{n=1}^{N} \kappa_{n}$ and $H(t)$ is the Heaviside step function. $\chi$ denotes the usual compressibility calculated with the formula $\chi=\left(c^{2} \rho\right)^{-1}$ which holds in liquids as well as in soft biological tissues of density $\rho$. Stokes' equation (2) is seen to be the special case of a single relaxation mechanism $N=1$, $\kappa_{1}=\chi$.

All three simulation methods produce waveforms in good agreement to the measured waveform. Of course, the approximation will become less accurate with longer propagation distance in the absorbing medium.

Simulation results may also be validated if we recall that the frequency domain transfer function of the pressure signal propagating through a layer with complex wavenumber $K(\omega)$ and thickness $d$ equals $\exp (\mathrm{iK}(\omega) d)$. So the simulated 'water-fat-water' waveform may be obtained from the measured 'water-only' waveform by inverting water attenuation over the fat thickness and then applying fat attenuation over the same fat thickness.

\section{Compensation of acoustic attenuation}

When taking acoustic attenuation into account, the wave equation, e.g. (2) or (3), is not invariant to time reversal any more. First order terms in time $t$ or higher order odd terms change sign if time is reversed. The equations then do not behave "well" any more, noise is amplified exponentially and regularization methods have to be used for solving these time reversed equations. Using such regularized methods the spatial resolution, that is limited by the frequency-dependent damping, is improved (Burgholzer et al., 2007). To prevent highfrequency noise from growing exponentially, Fourier spectral methods (Trefethen, 2000) in space have been used. They utilize the spatial Fourier transform to calculate the Laplacian and therefore allow for incorporating a damping of higher frequencies when calculating the time reversal. A similar algorithm to compensate acoustic attenuation step by step was proposed by (Treeby et al., 2010c).

In the second strategy, attenuation is compensated in one step where an approximation to the 'un-attenuated' measured signal is calculated from the attenuated measured signal by solving an appropriate integral equation (La Riviere et al., 2006); then an ordinary reconstruction algorithm for photoacoustic tomography is applied as in the absence of attenuation.

The Matlab Toolbox by Treeby et al. implements a state-of-the-art algorithm to simulate ultrasound wave propagation ('the direct problem') and image reconstruction ('the inverse problem') for PAI. For the direct and inverse problems arbitrary source distributions and detector geometries, variable medium densities and sound speeds and an arbitrary constant medium absorption are supported in 1D-3D. The algorithm is first-order finite difference in time and pseudospectral in space (working in spatial Fourier space = 'k-space'). The linearized Euler equations in a fluid $\frac{\partial \mathbf{u}}{\partial t}=-\frac{1}{\rho^{*}} \nabla p, \frac{\partial \rho}{\partial t}=-\rho^{*} \nabla \cdot \mathbf{u}$ together with an adiabatic equation of state $p=c_{0}^{2} \rho$ are solved up to time $T$ for the sound velocity vector $\mathbf{u}$, pressure $p$ and density $\rho$. In the case of absorbing media the equation of state is extended to 
$p(\mathbf{r}, t)=c_{0}^{2} \rho(\mathbf{r}, t)+\mathrm{F}^{-1}\left(\tau k^{y-2} \frac{\partial \hat{\rho}(\mathbf{k}, t)}{\partial t}+\eta k^{y-1} \hat{\rho}(\mathbf{k}, t)\right)$, where $\tau$ is related to attenuation and $\eta$ to dispersion (Treeby \& Cox, 2010) and the inverse Fourier transform (IFT) in $d$ spatial dimensions is defined by $f(\mathbf{r})=\mathrm{F}^{-1}(\hat{f}(\mathbf{k}))=(2 \pi)^{-d} \int_{R^{d}} \hat{f}(\mathbf{k}) \exp (i \mathbf{k} \cdot \mathbf{r}) d \mathbf{k}$. With this extension of the equation of state frequency-domain ultrasound absorption power laws of the form $\alpha(\omega)=\alpha_{0} \cdot|\omega|^{y}$ can be supported.

For the inverse problem, the same algorithm as for the direct problem is applied, but with zero initial conditions $p(\mathbf{r}, 0)=0, \mathbf{u}(\mathbf{r}, 0)=\mathbf{0}$ and time-varying Dirichlet boundary conditions on detector surface points $\mathbf{r}_{S}$ in the form of the time-reversed sensor data $p\left(\mathbf{r}_{S}, t\right)=p_{\text {mens }}\left(\mathbf{r}_{S}, T-t\right)$. Absorption is compensated by inverting the sign of $\tau$ but leaving $\eta$ unchanged. Additionally, a regularization filter is applied in $k$-space that suppresses the higher frequencies.
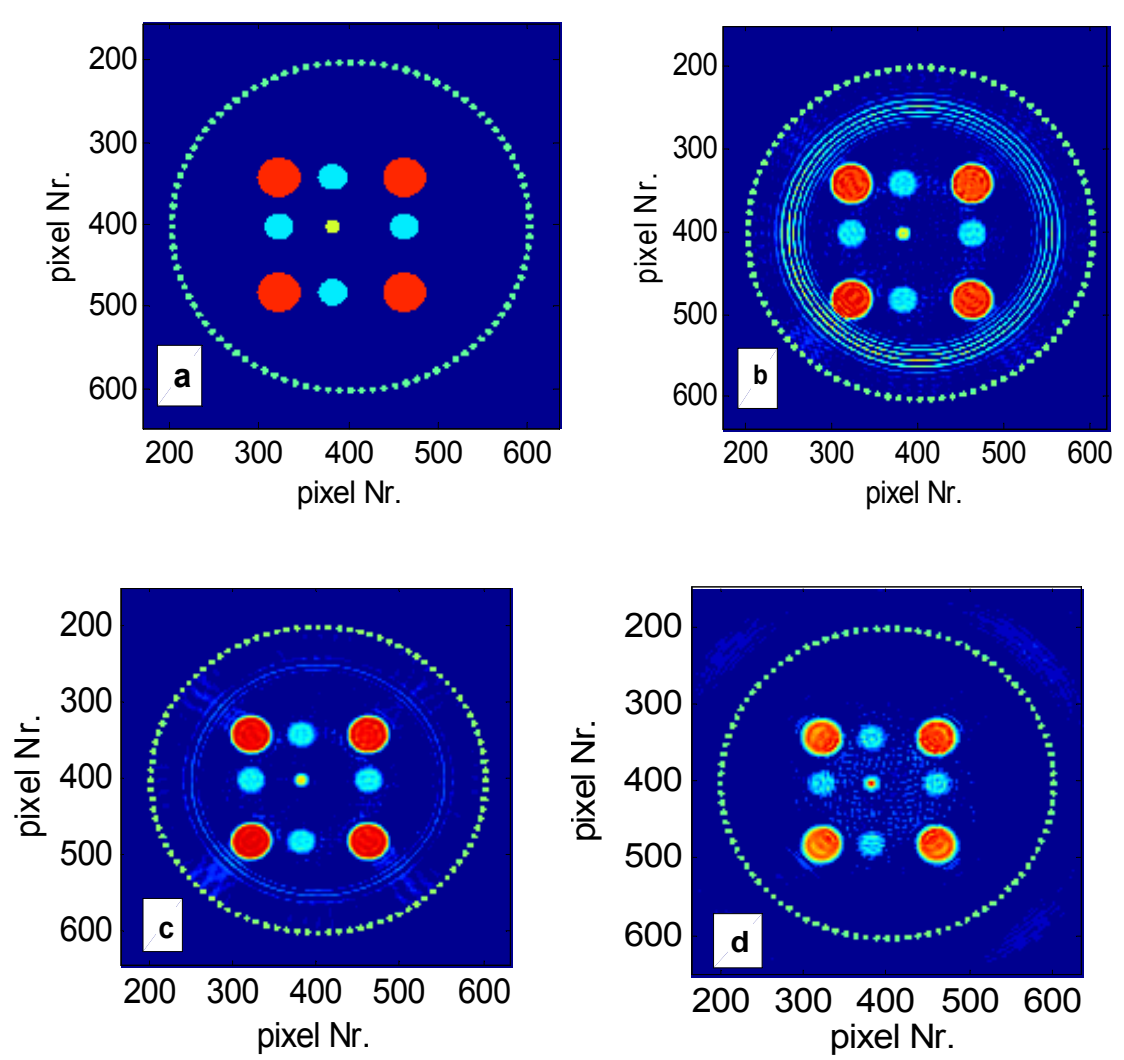

Fig. 5. Reconstruction of nine-circle phantom: (a) initial pressure distribution, (b) reconstruction Matlab toolbox with taper ratio $r=0.5$, (c) reconstruction Matlab toolbox with taper ratio $r=0.75,(d)$ reconstruction one-step compensation plus fast series algorithm 
A Tukey (tapered cosine) window is chosen for this filter. This window is parameterized by its cutoff-frequency in $k$-space (here $2 \pi \cdot 10^{4} \mathrm{rad} / \mathrm{m}$, corresponding to $2 \pi \cdot 15 \mathrm{MHz}$ in $\omega$ space) and its taper ratio $r$. The ratio controls the filter roll-off: raising $r$ suppresses more high-frequency content in the measurement noise. In Fig. 5a we display a complex phantom consisting of nine circles of different sizes and source strengths, surrounded by a circular array of detectors (which could represent line detectors in 3D). This phantom was reconstructed first with taper ratio $r=0.5$ (Fig. $5 b$ ) and then with taper ratio $r=0.75$ (Fig. $5 \mathrm{c})$. The absorption parameters were an exponent of $y=1.5$ and $\alpha_{0}=3 \mathrm{~dB} /\left(\mathrm{cm} \cdot \mathrm{MHz} z^{1.5}\right)$, and the 'measurement noise' consisted only of numerical noise.

Note that in Fig. $5 \mathrm{~b}$ ring-like artifacts are produced during reconstruction, which are much reduced in Fig. $5 \mathrm{c}$ because there the regularization filter is more restrictive. With taper ratio $r=1$ they disappear altogether. If a simpler phantom is used, e.g. just a single circle, taper ratio $r=0.5$ does not produce any artifacts. To sum up, complex, fine-structured objects exhibit more signal content and hence more measurement noise at higher frequencies. Then the regularization filter must be parameterized more restrictively than for compact objects.

The formula used for calculating and compensating attenuation in one step was first presented by (LaRiviere et al., 2006) and reads

$$
\tilde{p}_{\text {att }}\left(\mathbf{r}_{S}, \omega\right)=\frac{\omega}{c_{0} \cdot K(\omega)} \int_{-\infty}^{+\infty} p_{\text {ideal }}\left(\mathbf{r}_{S}, t\right) \exp \left(i c_{0} K(\omega) \cdot t\right) d t
$$

This relation yields the temporal Fourier transform of the attenuated pressure at a given point $\mathbf{r}=\mathbf{r}_{S}$ (and, via an IFT, the attenuated pressure itself) if the ideal (i.e. un-attenuated) pressure is known over time at that point. This can be exploited for the direct problem where the evaluation involves only two nested numerical integrations. For the inverse problem, the left-hand side of (4) is known and the relation represents an integral equation to be solved for $p_{\text {ideal }}\left(\mathbf{r}_{S}, t\right)$. Since this solution is very sensitive to measurement noise, i.e. noise in the spectrum of $p_{a t t}\left(\mathbf{r}_{s}, t\right)$, regularization is necessary, for instance in the form of truncated singular value decomposition (SVD, see e.g. La Riviere et al., 2006).

If the detector geometry is regular, fast reconstruction algorithms using series expansions in eigenfunctions of the Laplacian on the regular domain are available for the lossless case making the whole reconstruction with compensation much faster than reconstruction with the mentioned toolbox algorithm. In Fig. 5d we display a reconstruction of the nine-circle phantom using this procedure. To apply the SVD, a certain noise level in temporal Fourier space must be assumed. It is desirable to base this assumption on physical reasons - this will be discussed below. Here we assume a white Gaussian noise with a standard deviation of $0,1 \%$ of the largest amplitude in the spectrum.

Fig. $5 \mathrm{~d}$ shows that the reconstruction with the one-step compensation is almost of the same quality as the reconstruction with the Matlab toolbox algorithm. However, computation time is reduced by a factor of 10 in comparison to the toolbox algorithm.

We already observed that the quality of reconstructions depends crucially on a good analysis of the amplitude and spectral distribution of the measurement noise. On the source side, advance knowledge of the type of object to be imaged is helpful (more bulky and compact, or more fine-structured). On the detector side, noise is created by fundamental physical processes as well as by technological limitations. 
First, for any material volume $V$ at temperature $T$ pressure fluctuations of variance $\operatorname{Var}(p)=k_{B} T / \chi V \quad$ (Landau\&Lifshitz, 1980) will occur where $\chi$ is the adiabatic compressibility and $k_{B}$ is the Boltzmann constant. So if detectors are e.g. thin foils with small volume, this may play a role since noise amplitudes grow like $V^{-1 / 2}$.

Second, the fluctuation-dissipation theorem states that sound absorption processes in media create pressure fluctuations. If the absorption is described by Stokes' equation (2), a straightforward calculation yields a power spectral density of these pressure fluctuations $S_{P}(\omega)=\frac{2 k_{B} T}{\pi} \cdot \chi^{-2} \frac{\tau}{\left(1+\omega^{2} \tau^{2}\right)^{2}}$.

Third, noise is created by the analog-to-digital conversion process during electronic acquisition of the pressure signal. If this quantization noise is assumed to be white, its power spectral density at sampling frequency $f_{s}$ will be constant and equal $q^{2} / 12 f_{s}$ (Widrow \& Kollar, 2008) where $q$ is the quantization interval.

We have given only an outline of the considerations that have to be taken into account for a good estimation of the measurement noise. In a concrete application the relative importance of the mentioned contributions will depend on sizes and shapes of objects and detectors, the physics of the ultrasound propagation medium (compressibility, temperature) and the properties of the measurement devices.

\section{Stochastic processes for modeling acoustic attenuation}

As for any other dissipative process the energy of the attenuated acoustic wave is not lost but is transferred to heat, which can be described in thermodynamics by an entropy increase. This increase in entropy is equal to a loss of information, as defined by Shannon, and no compensation algorithm can compensate this loss of information. This is a limit given by the second law of thermodynamics. But can this limit be found in the algorithms for compensation of acoustic attenuation given in the previous section? Fluctuations of the measured pressure are "amplified" exponentially during the compensation and therefore we suspect a relation between the entropy production caused by acoustic attenuation and the fluctuations. Indeed such a relation is known in statistical physics as the fluctuationdissipation theorem and is due to (Callen \& Welton, 1951), (Callen \& Green, 1952), and (Greene \& Callen, 1952). It represents in fact a generalization of the famous (Johnson, 1928) (Nyquist, 1928) formula in the theory of electric noise.

In this section we use the theory of non-equilibrium thermodynamics presented by S.R. de Groot and P. Mazur (De Groot \& Mazur). More about random variables and stochastic processes can be found e.g. in the book about Statistical Physics from (Honerkamp, 1998). An introduction to stochastic processes on an elementary level has been published by. (Lemons, 2002), also containing "On the Theory of Brownian Motion" by (Langevin, 1908). An introduction to Markov Processes is given by (Gillespie, 1992).

In this section the measured pressure signal is treated as a time-dependent random variable with a mean value and a variance as a function of time. To be able to use the results of some "model" stochastic processes given in literature (Ornstein-Uhlenbeck process or damped harmonic oscillator) for a model of photoacoustic reconstruction we have changed the initial conditions: instead of a defined initial value (with zero variance) we have taken the stochastic process at equilibrium before time zero and at a time zero a certain perturbation has been applied to the process (e. g. a rapid change in momentum for the damped 
harmonic oscillator - called kicked damped oscillator in this chapter). Reconstruction of the size of this perturbation at time $t=0$ from the measurement after a time $t$ shows how the information about the size of this kick at $t=0$ gets lost with increasing time if dissipative processes occur. This change of the initial conditions has a significant advantage: it turns out that the variance stays constant in time, while the mean value is a function of time. This facilitates the calculations of the entropy production and of the information loss due to the stochastic process.

\section{Gauss-Markov processes and entropy}

In this section we shall assume that the time varying stochastic processes will have GaussMarkov character. In doing so we do not wish to assume that all dissipative macroscopic processes considered belong to this specific class of Gauss-Markov processes. It may, however, be surmised that a number of real phenomena may, with a certain approximation, be adequately described by such processes (De Groot \& Mazur). The advantage of specifying more precisely the nature of the processes considered is that it enables us to discuss, on the level of the theory of random processes, the behavior of entropy production and of information loss.

Following the theory of random fluctuations given e.g. by (De Groot \& Mazur), we take as a starting point equations analogous to the Langevin equation used to describe the velocity of a Brownian particle:

$$
\frac{d \mathbf{a}}{d t}=-\mathbf{M} \cdot \mathbf{a}+\boldsymbol{\varepsilon}(t)
$$

The components of the vector $\mathbf{\alpha}$ are the random variables $a_{i}(i=1,2, \ldots, n)$, having zero mean value at equilibrium. The matrix $\mathbf{M}$ of real phenomenological coefficients is independent of time. The vector $\varepsilon(t)$ represents white noise, which is uncorrelated at different times. The distribution density of a turns out to be an n-dimensional Gaussian distribution:

$$
f(\mathbf{\alpha}, t)=\frac{1}{\sqrt{(2 \pi)^{n}|\Sigma|}} e^{-\frac{1}{2}(\mathbf{\alpha}-\overline{\mathbf{\alpha}}(t))^{T} \Sigma^{-1}(\mathbf{\alpha}-\overline{\mathbf{\alpha}}(t))}
$$

with the mean value $\overline{\mathbf{a}}(t)$ and the covariance matrix $\Sigma$, which is usually also a function of time but for the initial conditions used later on the covariance matrix will be constant in time. $|\Sigma|$ is the determinant of the covariance matrix. If the initial value of $\mathbf{a}$ is given by a given $\overline{\mathbf{a}}_{0}$, the mean value at a later time $t$ will be:

$$
\overline{\mathbf{a}}(t)=e^{-\mathbf{M} t} \cdot \overline{\mathbf{a}}_{0}
$$

By an adequate coordinate transformation of $\mathbf{a}$ the matrix $\mathbf{M}$ can be diagonalized: the eigenvalues of $\mathbf{M}$ are the elements of the diagonal matrix.

According to the second law of thermodynamics the entropy of an adiabatically insulated system must increase monotonously until thermodynamic equilibrium is established within the system. Then the entropy will be set to zero and the entropy at a time $t$ is:

$$
S(t)=-k_{B} \int f(\mathbf{\alpha}, t) \ln \frac{f(\mathbf{\alpha}, t)}{f(\mathbf{\alpha}, t \rightarrow \infty)} d \mathbf{\alpha}
$$


with the Boltzmann constant $k_{B}$. For a constant covariance matrix the above integration results in:

$$
S(t)=-\frac{1}{2} k_{B} \overline{\mathbf{\alpha}}(t)^{T} \Sigma^{-1} \overline{\mathbf{\alpha}}(t)
$$

Before modeling the attenuated acoustic wave as a Gauss-Markov process we give two simple examples: the Orstein-Uhlenbeck process with only one component of $\mathbf{a}$ as a model for the velocity of a Brownian particle and the damped harmonic oscillator with two components of $\mathbf{\alpha}$.

\section{Example: kicked Ornstein-Uhlenbeck process}

If the random vector $\mathbf{a}$ in eq. (5) has only one component we get the Langevin equation

$$
\frac{d v(t)}{d t}=-\gamma \cdot v(t)+\sigma \eta(t)
$$

which was used to describe Brownian motion of a particle. The random variable $v$ is the particle velocity, $-\gamma \cdot v$ is the viscous drag, and $\sigma$ is the amplitude of the random fluctuations. The Langevin equation governs an Ornstein-Uhlenbeck process, after (Uhlenbeck \& Ornstein, 1930), who formalized the properties of this continuous Markov process. Now we assume that initially we have thermal equilibrium with zero mean velocity. At time zero the particle is kicked which causes an immediate change in velocity of $v_{0}$. Following eq. (7) the mean value $\bar{v}(t)$ shows an exponential decay:

$$
\bar{v}(t)=e^{-\gamma t} \cdot \bar{v}_{0}
$$

The variance of the velocity $\operatorname{Var}(v)$ is $\sigma^{2} / 2 \gamma$ and is constant in time. In Fig. 6 time and velocity are scaled to be dimensionless and the standard deviation (square root of the variance) of the velocity is normalized.

From eq. (9) the information loss equal to the entropy production till the time $t$ after the kick is:

$$
\Delta S(t)=k_{B} \frac{\gamma}{\sigma^{2}} \bar{v}(t)^{2}
$$

On the other hand the entropy production known from thermodynamics is the dissipated energy $\Delta Q$, which is the kinetic energy of the Brownian particle of mass $m$, divided by the temperature $\mathrm{T}$ :

$$
\Delta S(t)=\frac{\Delta Q}{T}=\frac{m \bar{v}(t)^{2}}{2 T}
$$

The thermodynamic entropy production in eq. (12) has to be equal to the loss of information in eq. (13), and therefore we get for the variance of the velocity:

$$
\frac{\sigma^{2}}{2 \gamma}=\frac{k T}{m}
$$


Eq. (14) has been derived previously by the equipartition theorem: the equilibrium energy associated with fluctuations in each degree of freedom is $k_{B} T / 2$. We have used the equity of entropy production and information loss. Eq. (14) states a connection between the strength of the fluctuations, given by $\sigma^{2}$, and the strength of the dissipation $\gamma$. This is the fluctuation-dissipation theorem in its simplest form for uncorrelated white noise.

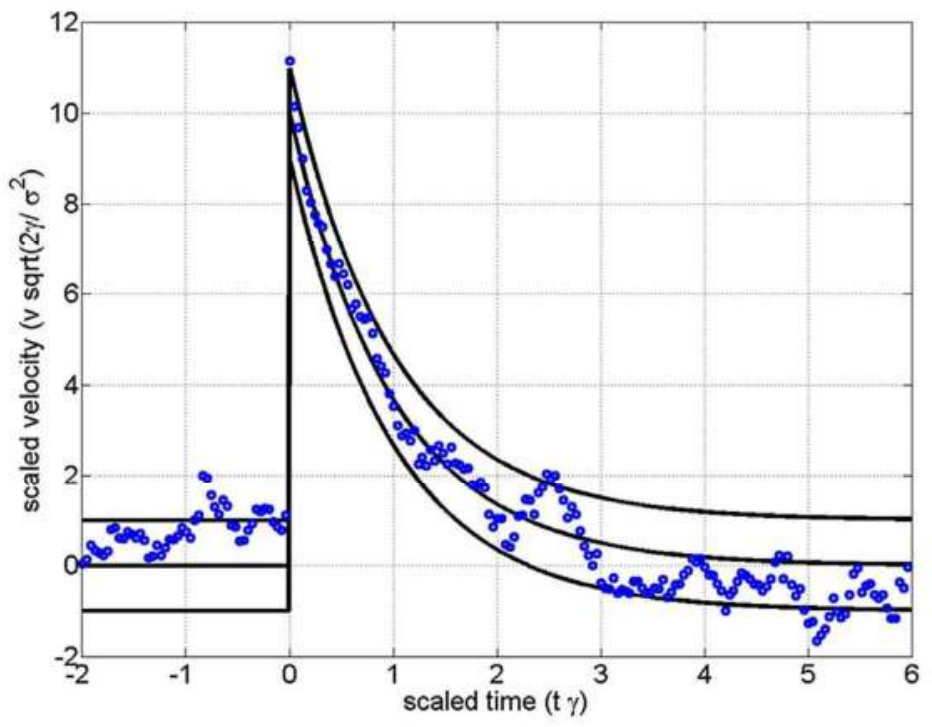

Fig. 6. Points on a sample path of the normalized kicked Ornstein-Uhlenbeck process defined by the Langevin eq. (10). The solid lines represent the mean, and mean \pm standard deviation of the scaled velocity coordinate. At the time $t=0$ a value of $\mathrm{v}_{0}=10$ has been added to the scaled velocity. After some time the information of the amplitude gets more and more lost due to the fluctuations

It is instructive to determine the least square estimator (Honerkamp, 1998) for the initial velocity $v_{0}$. If we write for the estimated initial velocity $v_{r}$

$$
v_{r}=R(t) \cdot v(t)
$$

we calculate $R(t)$ by minimizing the mean error $\left\langle\left(v_{0}-v_{r}\right)^{2}\right\rangle$ :

$$
R(t)=\frac{e^{-\gamma t}}{e^{-2 \gamma t}+\sigma^{2} / 2 \gamma v_{0}^{2}}
$$

This gives the Tikhonov regularization with $\sigma^{2} / 2 \gamma v_{0}{ }^{2}=\operatorname{Var}(v) / v_{0}{ }^{2}$ as regularization parameter. The inverse square root of the regularization parameter $v_{0} / \sqrt{\operatorname{Var}(v)}$ can be interpreted as signal-to-noise-ration (SNR) of $v(t)$.

\section{Example: kicked harmonic osciallator}

For modeling of acoustic waves one needs in addition to the dissipation also an oscillating term. For pure oscillation without damping we have no loss of information (Fig. 7). 


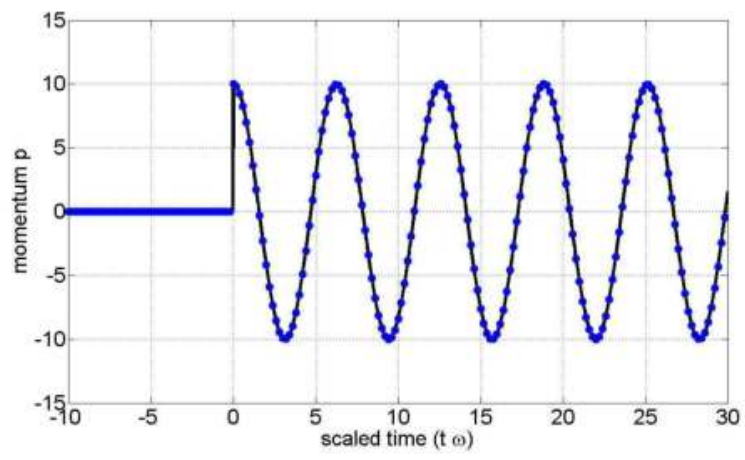

Fig. 7. Points on a sample path of the kicked harmonic oscillator without damping. The momentum used to kick the oscillator can be reconstructed without loss of information at any time after the kick if $\omega$ is known

The stochastically damped harmonic oscillator combines the oscillatory and the diffusive behavior and therefore it is a good starting point to model attenuated acoustic waves. The equations of motion are (using the momentum $p$ instead of the velocity $v$ ):

$$
\begin{gathered}
\frac{d x(t)}{d t}=\frac{1}{m} p(t) \\
\frac{d p(t)}{d t}=-\frac{\gamma}{m} \cdot p(t)-m \omega_{0}^{2} x+\sigma \eta(t)
\end{gathered}
$$

These equations can be combined using a two dimensional random vector $\mathbf{\alpha}=(x, p)$ and were solved already by (Chandrasekhar, 1943) for definite initial conditions $x(0)$ and $p(0)$. Again we have changed the initial conditions to an oscillator with zero mean values kicked by an initial momentum $p_{0}$. In Fig. 8 the damping is chosen to be $\gamma=m \omega_{0} / 3$.

Using the fluctuation-dissipation theorem $\sigma^{2} / 2 \gamma=k T$ one obtains for the distribution function

$$
f(x, p, t)=\frac{1}{2 \pi \frac{k T}{\omega_{0}}} e^{-\frac{1}{2 m k T}(p-\bar{p}(t))^{2}-\frac{1}{2 k T} m \omega_{0}^{2}(x-\bar{x}(t))^{2}}
$$

where $\bar{x}(t)$ and $\bar{p}(t)$ are the solutions of the ordinary (non-stochastic) damped harmonic oscillator. Then one gets for the information loss from eq. (9)

$$
S(t)=-\frac{1}{T}\left(\frac{1}{2} m \omega_{0}^{2} \bar{x}(t)^{2}+\frac{\bar{p}(t)^{2}}{2 m}\right)=-\frac{1}{T}\left(E_{p o t}+E_{k i n}\right) ，
$$

which is equal to the entropy from thermodynamics, where $E_{p o t}+E_{k i n}$ is the total energy of the harmonic oscillator (sum of the potential and kinetic energy). This fact confirms again that the fluctuation-dissipation theorem for the damped harmonic oscillator $\sigma^{2} / 2 \gamma=k_{B} T$ can be derived from the equity of entropy production and information loss. 

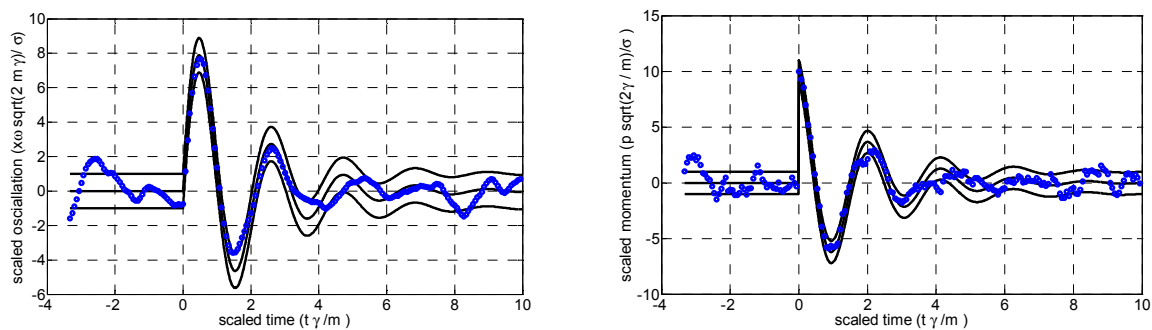

Fig. 8. Points on a sample path of the normalized kicked damped harmonic oscillator (eq. (17) and (18)). The solid lines represent the mean, and mean \pm standard deviation of the scaled oscillation (left) and momentum (right). At the time $\mathrm{t}=0$ a value of $\mathrm{p}_{0}=10$ has been added to the scaled momentum. After some time the information about the value of $\mathrm{p}_{0}$ gets more and more lost due to the fluctuations

For the mean value of the momentum $\bar{p}(t)$ one gets from eq. (7):

$$
\bar{p}(t)=p_{0}\left[\cos (\omega t)-\frac{\gamma}{2 m} \frac{\sin (\omega t)}{\omega}\right] e^{-\frac{\gamma t}{2 m}}
$$

Compared to the Ornstein-Uhlenbeck process (eq. (11)) the mean value has not only an exponential decay in time but also an oscillation with a frequency $\omega=\sqrt{\omega_{0}^{2}-\gamma^{2} /\left(4 m^{2}\right)}$. As mentioned above (Fig. 7) the oscillating term does not change the entropy and no information is lost. In the average only the exponential decay causes production of entropy and the information about the value of $p_{0}$ gets more and more lost due to the fluctuations. The entropy production which is proportional to the total energy is shown in Fig. 9.

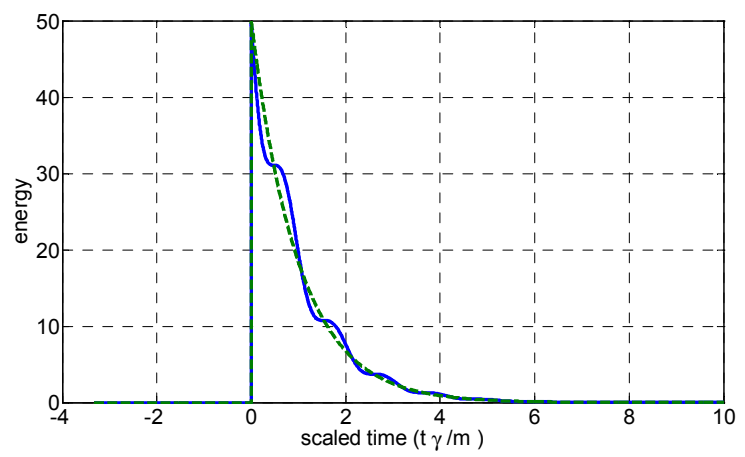

Fig. 9. Total energy of the damped harmonic oscillator (solid line) shows in the average an exponential decay with the time constant $\gamma / m$ (dashed line)

\section{Attenuated acoustic wave as a stochastic process}

In photoacoustic imaging, the laser pulse at a time $t=0$ generates an initial pressure distribution $p_{0}(\mathbf{r})$ (see section 2). For numerical calculations we use a discrete space $\mathbf{r}=\mathbf{r}_{j}$ $(j=1, . ., N)$, where $\mathbf{r}_{j}$ are $N$ points on a cubic lattice with a spacing of $\Delta r$ within the sample 
volume $V$. At a time $t$ the pressure distribution $p_{j}(t)$ can be represented by a Fourier series (Barret et al. 1995), including the time $t=0$ with the initial pressure distribution:

$$
p_{j}(t)=p\left(\mathbf{r}_{j}, t\right)=\sum_{k=1}^{N} \hat{p}_{k}(t) \varphi_{k}\left(\mathbf{r}_{j}\right) \quad \text { with } \varphi_{k}(\mathbf{r})=e^{2 \pi i \boldsymbol{\rho}_{k} \cdot \mathbf{r}} \cdot D(\mathbf{r})
$$

$D(\mathbf{r})$ is a support function which is one within the sample volume $V$ and zero outside. $\boldsymbol{\rho}_{k}$ are integer points on an infinite 3D lattices. From eq. (1) we get (if acoustic attenuation can be neglected):

$$
\hat{p}_{k}(t)=\cos \left(\omega_{k} t\right) \hat{p}_{k}(0) \quad \text { with } \omega_{k}^{2}=4 \pi^{2} c^{2} \boldsymbol{\rho}_{k}^{2} .
$$

Therefore we have only an oscillating term as shown in Fig. 7, but in higher dimensions, and no information is lost.

For an attenuated acoustic wave instead of the wave equation (1) we have used the Stokes equation (2) or the wave equation (3), giving for the spatial Fourier components $\hat{p}_{k}(t)$ not only an oscillating term but also an exponential decay:

$$
\hat{p}_{k}(t)=\left[A_{k} \sin \left(\omega_{k} t\right)+B_{k} \cos \left(\omega_{k} t\right)\right] e^{-\lambda_{k} t}
$$

where the phase factors $A_{k}$ and $B_{k}$ can be derived from the initial conditions and $\omega_{k}$ and $\lambda_{k}$ are functions of $\left|\boldsymbol{\rho}_{k}\right|$. Like for the damped harmonic oscillator this exponential decay causes the loss of information and can be modeled by a Gauss-Markov process. The information content in Fourier space is the same as in real space (Fig. 10). Therefore we can describe the information loss in the attenuated acoustic wave by the same model as for the damped harmonic oscillator, only in higher dimensions, as for each wave-vector-index $k$ an oscillator is needed.

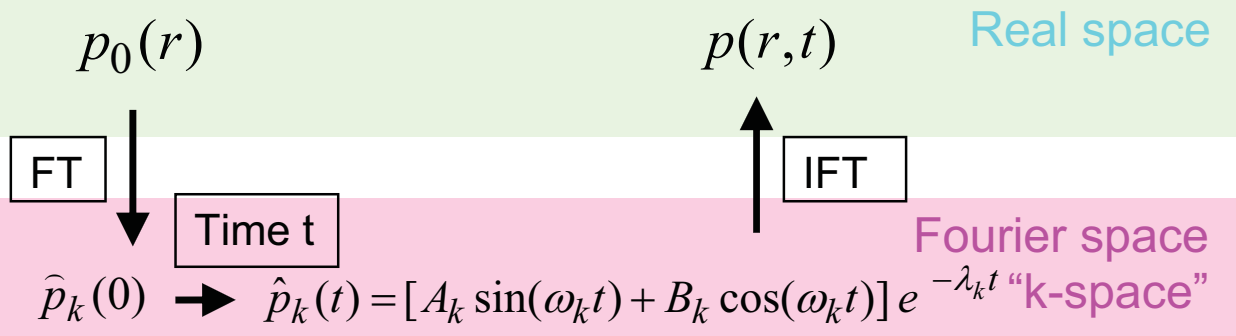

Fig. 10. The initial pressure distribution just after the laser pulse is Fourier transformed (FT). The time evolution of the Fourier series coefficients can be described similar to the mean value of a stochatsically damped harmonic oscillator. The pressure distribution after a time $t$ is then calculated by an inverse Fourier transform (IFT)

\section{One dimensional (1D) example: photoacoustic signal of a $0.2 \mathrm{~mm}$ thin layer in glycerin:}

The effect of acoustic attenuation for the reconstructed image is similar in 1D, 2D, and 3D (Burgholzer et al., 2010a and 2010b). As in 1D the reconstructed image is just the shifted 
measured signal, the effect of attenuation and of its compensation can be directly seen. The photoacoustic signal of a $0.2 \mathrm{~mm}$ thin absorbing layer in glycerin is calculated by using the scheme of Fig. 10 after a time of 4.5 microseconds. In glycerin the acoustic pressure can be described well by the Stokes' equation with a relaxation time of 244 picoseconds (Shutilov, 1988).

For an attenuated acoustic wave instead of the wave equation (1) we have used the Stokes equation (2) or the wave equation (3), giving for the spatial Fourier components $\hat{p}_{k}(t)$ not only an oscillating term but also an exponential decay:

Putting eq. (24) into the Stokes equation (2) one gets:

$$
\omega_{k}^{2}=4 \pi^{2} c^{2} \boldsymbol{\rho}_{k}^{2}\left(1-\frac{1}{4} c^{2} \boldsymbol{\rho}_{k}{ }^{2} \tau^{2}\right) \text { and } \lambda_{k}=\pi c^{2} \boldsymbol{\rho}_{k}{ }^{2} \tau
$$

The initial pressure distribution is a one dimensional square pulse corresponding to an absorbing layer of thickness $2 \mathrm{a}$ with $\mathrm{a}=0.1 \mathrm{~mm}$. In Fourier space we get:

$$
\hat{p}_{k}(0)=\frac{1}{\pi k} e^{-i k r} \sin (k a) w i t h k=2 \pi \rho_{k}
$$

The calculated signal after a time of 4.5 microseconds is shown in Fig. 11 (dashed line). The dashed dotted line shows the signal without attenuation (no relaxation time) and therefore represents the ideal reconstructed image.

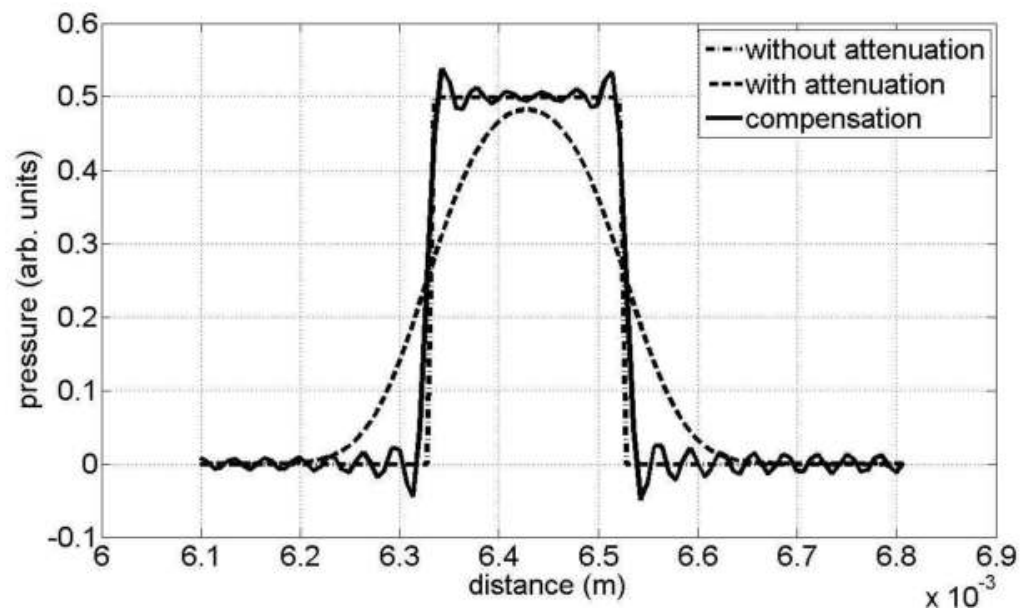

Fig. 11. Simulation result as an example for Stoke's equation: it is shown how an initial square pulse would look after a time of 4.5 microseconds in glycerine $(\tau=244 \mathrm{ps})$ (dashed line). The dashed dotted line shows the pulse without attenuation $(\tau=0)$, which correspond to the reconstruction of the initial pressure pulse, and the solid line is the SVD reconstruction of the initial pressure pulse (see text)

For compensation of the acoustic attenuation we use the time reversed process of the GaussMarkov process from Fig. 10. Similar to the stochastically damped oscillator the entropy production for the acoustic wave is set equal to the loss of information from the Gauss- 
Markov process with standard deviation $s_{k}$ and is approximated by an exponential decay in time (Fig. 9):

$$
\begin{gathered}
\Delta S(t) \approx \frac{1}{2} k_{B} \sum_{k} \frac{1}{S_{k}{ }^{2}} \exp \left(-2 \lambda_{k} t\right) \hat{p}_{k}(0)^{2} \\
\Delta S(t)=\frac{\Delta Q}{T} \approx \frac{1}{2 c_{0}{ }^{2} \rho_{0} T} \sum_{k} \frac{1}{s_{k}{ }^{2}} \exp \left(-2 \lambda_{k} t\right) \hat{p}_{k}(0)^{2}
\end{gathered}
$$

which gives a variance of $\hat{p}_{k}(t)$ in the spatial Fourier space ("k-space”) independent from $\mathrm{k}$ :

$$
s_{k}^{2}=k_{B} c_{0}^{2} \rho_{0} T
$$

In real space this gives the thermodynamic fluctuations. Or the other way around, the thermodynamic fluctuations have a quantity such that the information loss of the GaussMarkov process is equal to the dissipated heat divided by the temperature. Using the thermodynamic fluctuations for a detector size of $1 \mathrm{~cm}^{2}$ and the SVD regularization from section 4 the compensated $\hat{p}_{k}(t)$ is calculated. By applying a subsequent inverse Fourier transform (IFT) one gets the compensated pressure profile in real space (solid line in Fig. 11).

\section{Summary and conclusions}

Acoustic attenuation is modeled as a stochastic process: this helps to understand how thermodynamic entropy production and the decrease of information, which is "transported" in the acoustic wave, are closely connected on a microscopic scale. This theoretical insight enables to answer an important question in photoacoustic imaging: what is the highest possible compensation of attenuation and therefore the best spatial resolution one can achieve?

We could show that for thermal fluctuations the information loss of the reconstructed image is equal to the entropy production due to attenuation of the acoustic wave. Therefore it is sufficient to calculate the entropy production from the macroscopic mean values and it is not necessary to take the fluctuations of the pressure into account.

The size and locations of detectors in photoacoustic imaging should be optimized to get the best resolution and sensitivity. Up to now in such models it is not assumed that the pressure is a random variable, which favors small point like detectors. Taking thermal fluctuations and other noise into account will help to get a more realistic model for detectors and the reconstructed images from measured signals with these detectors.

\section{Acknowledgments}

This work has been supported by the Christian Doppler Research Association, by the Federal Ministry of Economy, Family and Youth, by the Austrian Science Fund (FWF) project numbers S10503-N20 and TRP102-N20, by the European Regional Development Fund (EFRE) in the framework of the EU-program Regio 13, and the federal state Upper Austria. 


\section{References}

Barret, H. H., Denny, J. L., Wagner, R. F. \& Myers, K. J. (1995). Objective assessment of image quality II, J. Opt. Soc. Am. A 12(5) 834-852

Bauer-Marschallinger, J., Berer, T., Roitner, H., Grün, H., Reitinger, B. \& Burgholzer, P. (2011). Ultrasonic attenuation of biomaterials for compensation in photoacoustic imaging, Proc. SPIE 7899, 789931

Bell, A. G. (1880). On the production and reproduction of sound by light: the photophone, American Journal of Science 20, 305-324

Berer, T., Hochreiner, A., Zamiri, S. and Burgholzer, P. (2010) Remote photoacoustic imaging on solid material using a two-wave mixing interferometer, Opt. Lett. 35, 4151-4153

Burgholzer, P., Hofer, C., Paltauf, G., Haltmeier, M. \& Scherzer, O. (2005). Thermoacoustic tomography with integrating area and line detectors, IEEE Trans. Ultrason. Ferroelectr. Freq. Control 52, 1577-1583

Burgholzer, P., Grün, H., Haltmeier, M., Nuster, R. \& Paltauf, G. (2007). Compensation of acoustic attenuation for high resolution photoacoustic imaging with line detectors, Proc. of SPIE Vol. 6437 643724-1

Burgholzer, P., Roitner, H., Bauer-Marschallinger, J. \& Paltauf, G. (2010a). Image Reconstruction in Photoacoustic Tomography using Integrating Detectors accounting for Frequency-Dependent Attenuation, Proc. of SPIE Vol. 7564 756423-1

Burgholzer, P., Berer, T., Gruen, H., Roitner, H., Bauer-Marschallinger, J., Nuster, R. \& Paltauf, G. (2010b). Photoacoustic Tomography using Integrating Line Detectors Invited, Journal of Physics: Conference Series, 214(1).

Callen, H. B. \& Welton, T. A. (1951), Phys. Rev. 83, 34

Callen, H. B. \& Greene, R. F. (1952). Phys. Rev. 86, 702

Chandrasekhar, S. (1943). Stochastic Problems in Physics and Astronomy, Reviews of Modern Physics 15, 1-89

De Groot, S.R. \& Mazur, P. (1984) Non-Equilibrium Thermodynamics, Dover Publications, Inc., New York

Gillespie, D. T. (1992). Markov Processes, Academic Press, New York

Greene, R. F. \& Callen, H. B. (1952). Phys. Rev. 88, 1387

Grün, H., Berer, T., Nuster, R., Paltauf, G. \& Burgholzer, P. (2010). Three-dimensional photoacoustic imaging using fiber-based line detectors, Journal of Biomedical Optics, 15(2), 021306-1 - 021306-8

Haltmeier, M., Scherzer, O., Burgholzer, P. \& Paltauf, G. (2004). Thermoacoustic computed tomography with large planar receivers, InverseProbl. 20, 1663-1673

Hansen, P. C. (1987). Rank-deficient and discrete ill-posed problems: Numerical aspects of linear inversion, SIAM, Philadelphia

Honerkamp, J. (1998). Statistical Physics, Springer-Verlag

Johnson, J. B. (1928). Thermal Agitation of Electricity in Conductors, Phy. Rev. 32, 97

Kiesel, S., Peters, K., Hassan, T. \& Kowalsky, M. (2007). Behavior of intrinsic polymer optical fibre sensor for large-strain, Meas. Sci.Technol. 18, 3144-3154

Landau, L. D. \& Lifshitz, E. M. (1980). Statistical physics, Part 1, Pergamon Press, Oxford

Langevin, P. (1908). Sur la théorie du mouvement brownien, Compets rendus Académie des Sciences (Paris) 146, 530-533. Translation by Anthony Gythiel, published in American Journal of Physics 65, 1079-1081 (1997) 
La Rivière, P. J., Zhang, J. \& Anastasio, M. A. (2006). Image reconstruction in optoacoustic tomography for dispersive acoustic media, Optics Letters. 31(6), 781-783

Lemons, D. S. (2002). An Introduction to Stochatic Processes in Physics, The Johns Hopkins University Press

Nachman, A. I., Smith III, J. F. \& Waag, R. C. (1990). An equation for acoustic propagation in inhomogeneous media with relaxation losses, J.Acoust.Soc.Am. 88, 1584-1595

Nuster, R., Gratt, S., Passler, K., Grün, H., Berer, T., Burgholzer, P. \& Paltauf, G. (2009). Comparison of optical and piezoelectric integration line detectors, in Biomedical Optics: Photons Plus Ultrasound: Imagingand Sensing 2009, edited by A. A. Oraevsky and L. H. Wang, Proc. SPIE 7177, 71770T

Nyquist, H. (1928). Phys. Rev. 32, 110

Paltauf, G., Nuster, R., Haltmeier, M. \& Burgholzer, P. (2006). Photoacoustic tomography using a Mach-Zehnder interferometer as acoustic line detector, Appl. Opt. 46, 3352-3358

Paltauf, G., Nuster, R., Passler, K., Haltmeier, M. \& Burgholzer, P. (2008). Optimizing Image Resolution in Three-Dimensional Photoacoustic Tomography With Line Detectors, in Biomedical Optics: Photons Plus Ultrasound: Imaging and Sensing 2008, Proc SPIE, 6856

Roitner, H. \& Burgholzer, P. (2011). Efficient modeling and compensation of ultrasound attenuation losses in photoacoustic imaging, Inverse Problems 27, 015003

Roitner, H., Bauer-Marschallinger, J., Berer, T. \& Burgholzer, P. (2011). Experimental Evaluation of ultrasound attenuation losses in photoacoustic imaging, submitted to J.Acoust.Soc.Am.

Royer, D. \& Dieulesaint, E. (2000). Elastic Waves in Solids I, Springer

Shutilov, V. A. (1988). Fundamental Physics of Ultrasound, Gordon and Breach Science Publishers

Stokes, G. G. (1845). On the theories of the internal friction of fluids in motion, and of the equilibrium and motion of elastic solids, Trans. Cambridge Philos. Soc. 8, 287-319

Szabo, T. L. (1994). Time domain wave equations for lossy media obeying a frequency power law, J.Acoust. Soc. Am. 96, 491-500

Treeby, B. E. \& Cox, B. T. (2010). Modeling power law absorption and dispersion for acoustic propagation using the fractional Laplacian, J.Acoust. Soc. Am. $127,2741-2748$

Treeby, B.E. \& Cox, B. T. (2010b). k-wave: Matlab toolbox for the simulation and reconstruction of photoacoustic wave fields, J. Biomed. Optics 15(2), 021314-1 - 021314-12

Treeby, B.E., Zhang, E. Z. \& Cox, B. T. (2010c). Photoacoustic tomography in absorbing acoustic media using time reversal, Inverse Problems 26, 115003

Trefethen, L. M. (2000). Spectral Methods in Matlab, SIAM, Philadelphia

Uhlenbeck, G. E. \& Ornstein, L. S. (1930). On the Theory of Brownian Motion, Phys. Rev. 36, $823-41$

Wang, L. V. (2008). Prospects of photoacoustic tomography, Medical Physics 35, 5758-5767

Widrow, B. \& Kollar, I. (2008). Quantization noise, Cambridge Books, Cambridge

$\mathrm{Xu}$, M. \& Wang, L. V. (2006). Photoacoustic imaging in biomedicine, Review of Scientific Instruments. 77(4), 041101-041122

Zhang, E. Z., Laufer, J. G., Pedley, R. B. \& Beard, P. C. (2009). In vivo high-resolution 3D photoacoustic imaging of superficial vascular anatomy, Phys. Med. Biol. 54, 1035-1046 


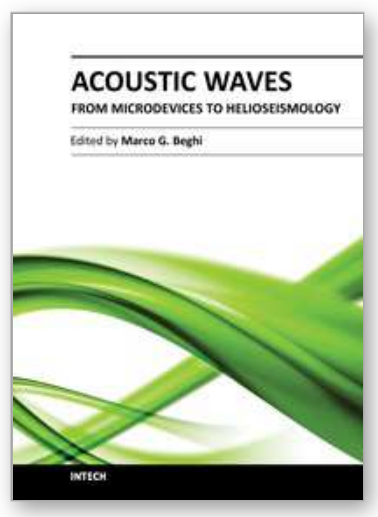

\author{
Acoustic Waves - From Microdevices to Helioseismology \\ Edited by Prof. Marco G. Beghi
}

ISBN 978-953-307-572-3

Hard cover, 652 pages

Publisher InTech

Published online 14, November, 2011

Published in print edition November, 2011

The concept of acoustic wave is a pervasive one, which emerges in any type of medium, from solids to plasmas, at length and time scales ranging from sub-micrometric layers in microdevices to seismic waves in the Sun's interior. This book presents several aspects of the active research ongoing in this field. Theoretical efforts are leading to a deeper understanding of phenomena, also in complicated environments like the solar surface boundary. Acoustic waves are a flexible probe to investigate the properties of very different systems, from thin inorganic layers to ripening cheese to biological systems. Acoustic waves are also a tool to manipulate matter, from the delicate evaporation of biomolecules to be analysed, to the phase transitions induced by intense shock waves. And a whole class of widespread microdevices, including filters and sensors, is based on the behaviour of acoustic waves propagating in thin layers. The search for better performances is driving to new materials for these devices, and to more refined tools for their analysis.

\title{
How to reference
}

In order to correctly reference this scholarly work, feel free to copy and paste the following:

P. Burgholzer, H. Roitner, J. Bauer-Marschallinger, H. Grün, T. Berer and G. Paltauf (2011). Compensation of Ultrasound Attenuation in Photoacoustic Imaging, Acoustic Waves - From Microdevices to Helioseismology, Prof. Marco G. Beghi (Ed.), ISBN: 978-953-307-572-3, InTech, Available from:

http://www.intechopen.com/books/acoustic-waves-from-microdevices-to-helioseismology/compensation-ofultrasound-attenuation-in-photoacoustic-imaging

\section{INTECH}

open science | open minds

\section{InTech Europe}

University Campus STeP Ri

Slavka Krautzeka 83/A

51000 Rijeka, Croatia

Phone: +385 (51) 770447

Fax: +385 (51) 686166

www.intechopen.com

\section{InTech China}

Unit 405, Office Block, Hotel Equatorial Shanghai

No.65, Yan An Road (West), Shanghai, 200040, China

中国上海市延安西路 65 号上海国际贵都大饭店办公楼 405 单元

Phone: +86-21-62489820

Fax: $+86-21-62489821$ 
(C) 2011 The Author(s). Licensee IntechOpen. This is an open access article distributed under the terms of the Creative Commons Attribution 3.0 License, which permits unrestricted use, distribution, and reproduction in any medium, provided the original work is properly cited. 